Pacific Journal of Mathematics

ON UNIVALENCE OF A CONTINUED FRACTION 


\section{ON UNIVALENCE OF A CONTINUED FRACTION}

\section{E. P. Merkes AND W. T. ScotT}

1. Introduction. For a fixed positive integer $\alpha$ let $K_{\alpha}$ denote the class of functions $f(z)$ which are regular at $z=0$ and which have $C$ fraction expansions of the form

$$
f(z) \sim \frac{z}{1}+\frac{a_{1} z^{\alpha}}{1}+\frac{a_{2} z^{\alpha}}{1}+\cdots+\frac{a_{n} z^{\alpha}}{1}+\cdots,\left|a_{n}\right| \leqq 1 / 4 .
$$

From an elementary convergence theorem for continued fractions [4, p.42], it follows that each function of the class $K_{\alpha}$ is regular for $|z|<1$. This and the one-to-one correspondence between $C$-fractions and power series $[4, \mathrm{p} .400]$ permit a replacement of the correspondence symbol in (1.1) by equality for $|z|<1$.

The purpose of this paper is to determine for $K_{\alpha}$ the radius of univalence, $U(\alpha)$, and bounds for the starlike radius, $S(\alpha)$, and the radius of convexity, $C(\alpha)$. In the case of $S$-fractions it was shown by Thale [3] that $U(1) \geqq 12 \sqrt{2}-16$ and Perron [2] established the fact that actual equality holds. This result is a special case of Theorem 2.1 whose proof employs value region techniques similar to those used by Thale and Perron. Moreover, the result $S(1) \geqq 8 / 9$ in [3] is improved in Theorem 4.2.

The developments in this depend on the following value region theorem which is an immediate consequence of a result of Paydon and Wall [1]:

THEOREM 1.1. If $f(z) \in K_{\alpha}$ and $|z|^{\alpha}=\rho^{\alpha} \leqq 4 r(1-r), 0 \leqq r \leqq 1 / 2$, then

$$
\left|\frac{f(z)}{z}-\frac{1}{1-r^{2}}\right| \leqq \frac{r}{1-r^{2}}
$$

Moreover, for $z=\sqrt[\alpha]{4 r(1-r)} e^{i m \pi / \alpha},(m=1,2, \cdots, \alpha)$, there is a value of $f(z) / z$ on the boundary of the disc (1.2) if and only if there exists a $\varphi, 0 \leqq \varphi<2 \pi$, such that $f(z) \equiv f(z ; \varphi)$, where

$$
f(z ; \phi)=\frac{z}{1}+\frac{\frac{1}{4} e^{i \varphi} z^{\alpha}}{1}+\frac{\frac{1}{4} z^{\alpha}}{1}+\cdots+\frac{\frac{1}{4} z^{\alpha}}{1}+\cdots .
$$

2. Determination of $U(\alpha)$. For $f(z) \in K_{\alpha}$ and for a fixed positive integer $n$ put

Received April 27, 1959, and in revised form July 31, 1959. 


$$
\begin{aligned}
f_{0, n}(z) & =z, \\
f_{p+1, n}(z) & =\frac{z}{1+a_{n-p} z^{\alpha-1} f_{p, n}(z)}, \quad(p=0,1, \cdots, n-1),
\end{aligned}
$$

where the numbers $a_{j}$ are the coefficients in the $C$-fraction expansion (1.1) of $f(z)$. It is easily seen that $f_{n, n}(z)$ is the approximant of (1.1) of order $n+1$, and that $f_{p, n}(z) \in K_{\alpha}$ for each $p$.

For non-negative integers $s, t$, and for non-zero numbers $z_{1}, z_{2},(2.1)$ may be used to show that

$$
\begin{aligned}
& z_{1}^{s} z_{2}^{t} f_{p+1, n}\left(z_{1}\right)-z_{1}^{t} z_{2}^{s} f_{p+1, n}\left(z_{2}\right) \\
= & \frac{f_{p+1, n}\left(z_{1}\right) f_{p+1, n}\left(z_{2}\right)}{z_{1} z_{2}}\left\{z_{1}^{s+1} z_{2}^{t}-z_{1}^{t} z_{2}^{s+1}-a_{n-p}\left[z_{1}^{t+\alpha-1} z_{2}^{s+1} f_{p, n}\left(z_{1}\right)\right.\right. \\
- & \left.\left.z_{1}^{s+1} z_{2}^{t+\alpha-1} f_{p, n}\left(z_{2}\right)\right]\right\}, \quad(p=0,1, \cdots, n-1) .
\end{aligned}
$$

This identity plays a fundamental role in the proof of the following theorem.

THEOREM 2.1. The radius of univalence of $K_{\alpha}$ is given by

$$
\begin{gathered}
U(2)=2 \sqrt{2} / 3, \\
{[U(\alpha)]^{\alpha}=\left[\frac{6 \sqrt{\alpha^{2}-2 \alpha+9}-2(\alpha+7)}{(\alpha-2)^{2}}\right], \quad(\alpha=1,3,4, \cdots) .}
\end{gathered}
$$

There is no larger region, containing the disc $|z|<U(\alpha)$, in which all functions of $K_{\alpha}$ are univalent.

Proof. For $f(z) \in K_{\alpha}$ and for a fixed positive odd integer $n=2 m+1$ it follows from (2.2) that

$$
\begin{aligned}
& f_{n, n}\left(z_{1}\right)-f_{n, n}\left(z_{2}\right) \\
= & \frac{f_{n, n}\left(z_{1}\right) f_{n, n}\left(z_{2}\right)}{z_{1} z_{2}}\left\{z_{1}-z_{2}-a_{1}\left[z_{1}^{\alpha-1} z_{2} f_{n-1, n}\left(z_{1}\right)-z_{1} z_{2}^{\alpha-1} f_{n-1, n}\left(z_{2}\right)\right]\right\} .
\end{aligned}
$$

Repeated application of (2.2) yields

$$
\begin{aligned}
& a_{1}\left[z_{1}^{\alpha-1} z_{2} f_{n-1, n}\left(z_{1}\right)-z_{1} z_{2}^{\alpha-1} f_{n-1, n}\left(z_{2}\right)\right] \\
= & \sum_{j=1}^{m+1}\left(z_{1} z_{2}\right)^{(j-1) \alpha+1}\left(z_{1}^{\alpha-1}-z_{2}^{\alpha-1}\right) \prod_{p=1}^{2 j-1} a_{p} \frac{f_{n-p, n}\left(z_{1}\right) f_{n-p, n}\left(z_{2}\right)}{z_{1} z_{2}} \\
- & \sum_{j=1}^{m}\left(z_{1} z_{2}\right)^{j \alpha}\left(z_{1}-z_{2}\right) \prod_{p=1}^{2 j} a_{p} \frac{f_{n-p, n}\left(z_{1}\right) f_{n-p, n}\left(z_{2}\right)}{z_{1} z_{2}} .
\end{aligned}
$$

For $z_{1}$ and $z_{2}$ in the disc $|z|<1, r$ can be chosen with $0<r<1 / 2$ such that $\left|z_{i}\right|^{\alpha} \leqq 4 r(1-r),(i=1,2)$, and by Theorem $1.1,\left|f_{p, n}\left(z_{i}\right) / z_{i}\right| \leqq 1 /(1-r)$, $(i=1,2 ; p=0,1, \cdots, n)$. When the triangle inequality is applied to. the right member of (2.5) and the indicated bounds are used, there 
results

$$
\begin{aligned}
& \left|\alpha_{1}\right|\left|z_{1}^{\alpha-1} z_{2} f_{n-1, n}\left(z_{1}\right)-z_{1} z_{2}^{\alpha-1} f_{n-1, n}\left(z_{2}\right)\right| \\
\leqq & \left|z-z_{2}\right|\left[\sum_{j=1}^{m+1}(\alpha-1)\left(\frac{r}{1-r}\right)^{2 j-1}+\sum_{j=1}^{m}\left(\frac{r}{1-r}\right)^{2 j}\right] \\
< & \left|z_{1}-z_{2}\right| \frac{r}{1-2 r}[\alpha-1-(\alpha-2) r] .
\end{aligned}
$$

This inequality and (2.4) give

$$
\begin{aligned}
& \left|f_{n, n}\left(z_{1}\right)-f_{n, n}\left(z_{2}\right)\right| \\
\geqq & \frac{\left|f_{n, n}\left(z_{1}\right) f_{n, n}\left(z_{2}\right)\right|}{\left|z_{1} z_{2}\right|}\left|z_{1}-z_{2}\right|\left\{1-\frac{r[\alpha-1-(\alpha-2) r]}{1-2 r}\right\} .
\end{aligned}
$$

Since Theorem 1.1 shows that neither of the factors $\left|f_{n, n}\left(z_{i}\right) / z_{i}\right|,(i=1,2)$, is zero, it follows from (2.6) that $f_{n, n}\left(z_{1}\right) \neq f_{n, n}\left(z_{2}\right)$ for $z_{1} \neq z_{2}$ if $r$ is such that $1-2 r>r[\alpha-1-(\alpha-2) r]$. This is equivalent to the condition $r<r_{0}(\alpha)$ where

$$
\begin{gathered}
r_{0}(2)=1 / 3 \\
r_{0}(\alpha)=\frac{\alpha+1-\sqrt{\alpha^{2}-2 \alpha+9}}{2(\alpha-2)}, \quad(\alpha=1,3,4, \cdots)
\end{gathered}
$$

and it is easily seen that $f_{2 m+1,2 m+1}(z)$ is univalent for $|z|^{\alpha}<[U(\alpha)]^{\alpha}=$ $4 r_{0}(\alpha)\left[1-r_{0}(\alpha)\right]$.

If the function $f(z)$ has a non-terminating $C$-fraction (1.1), the univalence of $f(z)$ for $|z|<U(\alpha)$ is an immediate consequence of the fact that $f(z)$ is the uniform limit of its sequence of even approximants, $f_{2 m+1,2 m+1}(z)$, for $|z| \leqq \rho<1$. The case where $f(z)$ has a $C$-fraction expansion (1.1) terminating with an odd number of partial quotients may be reduced to the previously considered case for even approximants by adding a partial quotient, $a_{2 m} z^{\alpha} / 1$ with $a_{2 m}=0$, and noting that $f_{2 m-1,2 m-1}(z)=$ $f_{2 m, 2 m}(z)$ in this case.

In order to complete the proof that the radius of univalence of $K_{\alpha}$ is the value $U(\alpha)$ given in (2.3), it suffices to exhibit a function of $K_{\alpha}$ which is not univalent in $|z|<\rho$ for any $\rho>U(\alpha)$. Such a function is the function $f(z, \pi)$ of $(1.3)$, that is,

$$
f(z, \pi)=\frac{2 z}{3-\sqrt{1+z^{\alpha}}}
$$

where the branch of the radical with positive real part for $|z|<1$ is used. This function is not univalent at the points $e^{i m \pi / \alpha} U(\alpha),(m=$ $1,2, \cdots, \alpha)$, where its derivative vanishes.

The final statement in Theorem 2.1 may be verified by applying to the function $f(z, \pi)$ the observation that, for every real $\theta, e^{-i \theta} f\left(e^{i \theta} z\right) \in K_{\alpha}$ 
whenever $f(z) \in K_{\alpha}$.

3. A covering theorem. The value region inequality (1.2) can be rewritten as

$$
\left|\frac{f(z)}{z}-\frac{4}{2+\rho^{\alpha}+2 \sqrt{1-\rho^{\alpha}}}\right| \leqq \frac{2\left(1-\sqrt{1-\rho^{\alpha}}\right)}{2+\rho^{\alpha}+2 \sqrt{1-\rho^{\alpha}}},
$$

where $|z|=\rho$ and $f(z) \in K_{\alpha}$. Thus for $|z|=\rho$ the following inequalities, which provide a means of comparison between $K_{\alpha}$ and various classes of univalent functions, are obtained:

$$
\begin{gathered}
\frac{2}{3-\sqrt{1-\rho^{\alpha}}} \leqq \Re\left\{\frac{f(z)}{z}\right\} \leqq \frac{2}{1+\sqrt{1-\rho^{\alpha}}}, \\
\left|\mathfrak{\Im m} \frac{f(z)}{z}\right| \leqq \frac{2\left(1-\sqrt{\left.1-\rho^{\alpha}\right)}\right.}{2+\rho^{\alpha}+2 \sqrt{1-\rho^{\alpha}}}, \\
\frac{2 \rho}{3-\sqrt{1-\rho^{\alpha}}} \leqq|f(z)| \leqq \frac{2\left(1-\sqrt{\left.1-\rho^{\alpha}\right)}\right.}{\rho^{\alpha-1}} \\
\left|\arg \frac{f(z)}{z}\right| \leqq \arcsin \frac{1-\sqrt{1-\rho^{\alpha}}}{2}
\end{gathered}
$$

Each of the inequalities (3.2)-(3.5) is sharp. This fact follows at once from Theorem 1.1 since equality in any one of (3.2)-(3.5) depends on the attainment by $f(z) / z$ of a suitable boundary value for the disc (3.1) or (1.2).

The following theorem is an immediate consequence of (3.4) and Theorem 2.1:

THEOREM 3.1. If $f(z) \in K_{\alpha}$, then the image of $|z|<U(\alpha)$ by $w=f(z)$ contains the disc

$$
|w|<\frac{2 U(\alpha)}{3-\sqrt{1-[U(\alpha)]^{\alpha}}},
$$

and is contained in the disc

$$
|w|<2 \frac{1-\sqrt{1-[U(\alpha)]^{\alpha}}}{[U(\alpha)]^{\alpha-1}} .
$$

These results are sharp.

4. A lower bound for $S(\alpha)$. An upper bound for $S(\alpha)$, the starlike radius for the class $K_{\alpha}$, is evidently the value $U(\alpha)$ determined in $\S 2$. In this section a lower bound for $S(\alpha)$ is found by determining a number 
$\rho_{1}(\alpha)$ such that every function of $K_{\alpha}$ is starlike in the disc $|z|<\rho_{1}(\alpha)$.

LEMMA 4.1. If $f(z) \in K_{\alpha}$ and $|a| \leqq 1 / 4$, then

$$
w(z)=-\frac{a z^{\alpha-1} f(z)}{1+a z^{\alpha-1} f(z)}
$$

satisfies

$$
\left|w-\frac{r^{2}}{1-r^{2}}\right| \leqq \frac{r}{1-r^{2}}
$$

whenever $|z|^{\alpha} \leqq 4 r(1-r), 0 \leqq r \leqq 1 / 2$.

Proof. The lemma is obvious when $a=0$. For $0<|a| \leqq 1 / 4$, (4.1) yields

$$
\frac{f(z)}{z}=\frac{1}{a z^{\alpha}} \cdot \frac{-w(z)}{1+w(z)},
$$

and the desired result is easily obtained by applying the inequality $|f(z) / z| \leqq 1 /(1-r)$, which is a consequence of Theorem 1.1.

LEMma 4.2. If $\alpha$ is a positive integer and if for fixed $r, 0<r<1 / 2$, $c$ and $d$ are numbers such that

$$
0 \leqq c \leqq \frac{1+(\alpha-2) r^{2}}{1-2 r^{2}}, 0<d=\frac{1+(\alpha-2) r}{1-2 r}-c,
$$

then $\sigma=1$ satisfies

$$
|\sigma-c| \leqq d
$$

Moreover, if $w$ is a parameter satisfying (4.2) and if $\sigma_{0}$ satisfies (4.4), then $\sigma_{1}$ satisfies (4.4) where

$$
\sigma_{1}=1+w\left(\sigma_{0}+\alpha-1\right) .
$$

Proof. It is obvious that $1-c \leqq d$ holds for all $r, 0<r<1 / 2$, and that $-d \leqq 1-c$ holds provided

$$
c \leqq \frac{2+(\alpha-4) r}{2(1-2 r)} .
$$

The fact that $\sigma=1$ satisfies (4.4) may be verified by noting that the upper bound of $c$ in this last inequality exceeds the upper bound on $c$ in (4.3) for all $r, 0<r<1 / 2$.

The proof of the second statement is obtained by using (4.2), (4.3), 
(4.4), (4.5), and the triangle inequality to show that

$$
\begin{aligned}
\left|\sigma_{1}-c\right| \leqq & \left|1-c+\frac{(c+\alpha-1) r^{2}}{1-r^{2}}\right| \\
& +(c+\alpha-1)\left|w-\frac{r^{2}}{1-r^{2}}\right|+|w|\left|\sigma_{0}-c\right| \\
\leqq & \frac{1+(\alpha-2) r^{2}-\left(1-2 r^{2}\right) c}{1-r^{2}}+\frac{(c+\alpha-1) r}{1-r^{2}}+\frac{r d}{1-r^{2}}=d .
\end{aligned}
$$

LEMMA 4.3. If (4.3) holds for $0<r<1 / 2$, there is a value of $c$ satisfying $c \geqq d$ if and only if $0<r \leqq r_{1}(\alpha)$, where $r_{1}(\alpha)$ is the smallest positive root of

$$
1-(\alpha+2) r+2(\alpha-1) r^{2}-2(\alpha-2) r^{3}=0 .
$$

Proof. By (4.3) the inequality $c \geqq d$ holds if and only if

$$
\frac{1+(\alpha-2) r^{2}}{1-2 r^{2}} \geqq \frac{1+(\alpha-2) r}{2(1-2 r)},
$$

which is equivalent to the statement that the left member of (4.6) is nonnegative. Clearly $r_{1}(\alpha)<1 / 2$.

THeOREM 4.1. If $f(z) \in K_{\alpha}$ and $c, d$ satisfy (4.3), where $|z|^{\alpha}=$ $\rho^{\alpha} \leqq 4 r(1-r)$, then

$$
\left|z \frac{f^{\prime}(z)}{f(z)}-c\right| \leqq d
$$

Proof. For the functions $f_{p, n}(z)$ of (2.1) put

$$
\sigma_{p, n}=z \frac{f_{p, n}^{\prime}}{f_{p, n}}, \quad w_{p, n}=-\frac{a_{n-p} z^{\alpha-1} f_{p+1, n}}{1+a_{n-p} z^{\alpha-1} f_{p+1, n}},
$$

and note by differentiation that $\sigma_{p+1, n}=1+w_{p, n}\left(\sigma_{p, n}+\alpha-1\right)$. For $|z|=\rho$ inductive application of Lemmas 4.1 and 4.2 shows that (4.7) holds for $f_{n, n}$, and the validity of (4.7) in this case for $|z| \leqq \rho$ follows from the maximum property for harmonic functions. Inasmuch as $f_{n, n}$ is the $(n+1)$ th approximant of (1.1) the theorem holds for functions of $K_{\alpha}$ having terminating $C$-fraction expansions. The validity of the theorem in the case of non-terminating $C$-fractions (1.1) is an immediate consequence of the uniform convergence of $f_{n, n}$ to $f$ on any closed subset of $|z|<1$.

THEOREM 4.2. The starlike radius of $K_{\alpha}$ satisfies $S(\alpha) \geqq \rho_{1}(\alpha)$ where 
$\left[\rho_{1}(\alpha)\right]^{\alpha}=4 r_{1}(\alpha)\left[1-r_{1}(\alpha)\right]$ and where $r_{1}(\alpha)$ is the smallest positive root of (4.6).

Proof. For $r \leqq r_{1}(\alpha)$ Lemma 4.3 shows that Theorem 4.1 can be applied to any function $f(z) \in K_{\alpha}$ with $c \geqq d$, and hence that

$$
\Re e z \frac{f^{\prime}(z)}{f(z)} \geqq 0, \quad|z| \leqq \rho_{1}(\alpha)
$$

Since this inequality insures that $f(z)$ is starlike for $|z|<\rho_{1}(\alpha)$ the proof is complete.

In particular, $r_{1}(1)=(\sqrt{3}-1) / 2$ and $S(1) \geqq 4 \sqrt{3}-6$ which improves the lower bound of $8 / 9$ obtained for $S(1)$ in [3].

5. A lower bound for $C(\alpha)$. It is clear that $S(\alpha)$ and $U(\alpha)$ are upper bounds for $C(\alpha)$, the radius of convexity of $K_{\alpha}$. In this section a lower bound for $C(\alpha)$ is found by determining a number $\rho_{2}(\alpha)$ such that every function of $K_{\alpha}$ is convex for $|z|<\rho_{2}(\alpha)$.

Lemma 5.1. Let $\alpha$ denote a positive integer and let $r_{2}(\alpha)$ be the smallest positive root of the equation:

$$
\begin{gathered}
1-\left(\alpha^{2}+2 \alpha+6\right) r+6\left(\alpha^{2}+\alpha+2\right) r^{2}-4\left(3 \alpha^{2}+2\right) r^{3} \\
+12(\alpha-1) \alpha r^{4}-4 \alpha(\alpha-2) r^{5}=0 .
\end{gathered}
$$

If for fixed $r, 0<r \leqq r_{2}(\alpha), \sigma_{0}$ and $\sigma_{1}$ are numbers which satisfy

$$
\left|\sigma_{0}-c\right| \leqq d,\left|\sigma_{1}-c\right| \leqq d,
$$

where

$$
\frac{1+(\alpha-2) r}{2(1-2 r)} \leqq c \leqq \frac{1+(\alpha-2) r^{2}}{1-2 r^{2}}, d=\frac{1+(\alpha-2) r}{1-2 r}-c,
$$

and if

$$
\gamma_{1}=2\left(\sigma_{1}-1\right)+\frac{\sigma_{1}-1}{\sigma_{1}}\left[\gamma_{0} \frac{\sigma_{0}}{\sigma_{0}+\alpha-1}+(\alpha-1) \frac{2 \sigma_{0}+\alpha-2}{\sigma_{0}+\alpha-1}\right],
$$

then $\left|\gamma_{0}\right| \leqq 1$ implies $\left|\gamma_{1}\right| \leqq 1$.

Proof. For $0<r<r_{1}(\alpha)$, where $r_{1}(\alpha)$ is as determined in Theorem $4.2,0<d<c$ and

$$
c^{2}-d^{2}-c \leqq-\frac{\alpha r^{2}\left[(\alpha-1)-2(\alpha-2) r+2(\alpha-2) r^{2}\right]}{(1-2 r)^{2}\left(1-2 r^{2}\right)} \leqq 0 .
$$

Thus by (5.2) 


$$
\left|\frac{\sigma_{1}-1}{\sigma_{1}}-\frac{c^{2}-d^{2}-c}{c^{2}-d^{2}}\right| \leqq \frac{d}{c^{2}-d^{2}}
$$

and it follows that

$$
\left|\frac{\sigma_{1}-1}{\sigma_{1}}\right| \leqq \frac{1}{c-d}-1
$$

Similarly, (5.2) can be used to show that

$$
\begin{gathered}
\left|\frac{\sigma_{0}}{\sigma_{0}+\alpha-1}\right| \leqq \frac{c+d}{c+d+\alpha-1}, \\
\left|(\alpha-1) \frac{2 \sigma_{0}+\alpha-2}{\sigma_{0}+\alpha-1}\right| \leqq(\alpha-1) \frac{2(c+d)+\alpha-2}{c+d+\alpha-1} .
\end{gathered}
$$

For $\left|\gamma_{0}\right| \leqq 1$ application to (5.4) of the triangle inequality, (5.2) and the bounds determined above lead to the inequality

$$
\left|\gamma_{1}\right| \leqq 2(c+d-1)+\left[\frac{1}{c-d}-1\right] \frac{(2 \alpha-1)(c+d)+(\alpha-1)(\alpha-2)}{c+d+\alpha-1}
$$

The desired inequality, $\left|\gamma_{1}\right| \leqq 1$, will hold for those values of $r<r_{1}(\alpha)$ for which the right member of (5.5) does not exceed 1, or equivalently, for which

(5.6) $c-d$

$$
\geqq \frac{(2 \alpha-1)(c+d)+(\alpha-1)(\alpha-2)}{(2 \alpha-1)(c+d)+(\alpha-1)(\alpha-2)+[3-2(c+d)][c+d+\alpha-1]}=D .
$$

Since $2 c=(c+d)+(c-d)$, (5.3) shows that the existence of a value of $c$ satisfying (5.6) is insured for all $r<r_{1}(\alpha)$ for which

$$
2 \frac{1+(\alpha-2) r^{2}}{1-2 r^{2}} \geqq(c+d)+D .
$$

This last inequality is equivalent to the requirement that the polynomial in the left member of (5.1) be non-negative.

The proof of the lemma will be completed by establishing the existence of a smallest positive zero, $r_{2}(\alpha)$ of (5.1) for which $r_{2}(\alpha)<r_{1}(\alpha)$. Since the equation (4.7) determining $r_{1}(\alpha)$ is equivalent to

$$
2 \frac{1+(\alpha-2) r^{2}}{1-2 r^{2}}=c+d
$$

and since $D>0$ for $r=r_{1}(\alpha)$, it follows that (5.7) fails to hold for $r=r_{1}(\alpha)$. The desired conclusion about $r_{2}(\alpha)$ is then easily obtained by noting that (5.7) holds with strict inequality for $r=0$. 
THOREM 5.1. The radius of convexity of $K_{\alpha}$ satisfies

$$
[C(\alpha)]^{\alpha} \geqq 4 r_{2}(\alpha)\left[1-r_{2}(\alpha)\right]=\left[\rho_{2}(\alpha)\right]^{\alpha}
$$

where $r_{2}(\alpha)$ is the smallest positive root of (5.1)

Proof. For the functions $f_{p, n}(z)$ of (2.1) put

$$
\sigma_{p, n}=z \frac{f_{p, n}^{\prime}}{f_{p, n}}, \quad \gamma_{p, n}=z \frac{f_{q, n}^{\prime \prime}}{f_{p, n}^{\prime}}
$$

It is easily verified from (2.1) that

$$
\gamma_{p+1}=2\left(\sigma_{p+1}-1\right)+\frac{\sigma_{p+1}-1}{\sigma_{p+1}}\left[\frac{\gamma_{p} \sigma_{p}}{\sigma_{p}+\alpha-1}+(\alpha-1) \frac{2 \sigma_{p}+\alpha-2}{\sigma_{p}+\alpha-1}\right]
$$

where the subscript $n$ has been omitted. Theorem 4.1 and the fact that $\gamma_{0, n}=0$ show that the hypotheses of Lemma 5.1 are satisfied, and inductive application of the lemma yields $\left|\gamma_{n, n}\right| \leqq 1$. It follows that

$$
\Re e\left[1+\gamma_{n, n}\right] \geqq 0,|z| \leqq \rho_{2}(\alpha),
$$

which insures the convexity of the $(n+1)$ th approximant of any $C$-fraction (1.1) for $|z|<\rho_{2}(\alpha)$, and the proof of the theorem may be completed, as in Theorem 4.1, by reference to uniform convergence.

It is found that $\rho_{2}(1)>.641$. An upper bound for $C(\alpha)$ can be obtained by finding for the function $f(z, \pi)$ of (1.3) the zeros of $z f^{\prime \prime}(z, \pi)+$ $f^{\prime}(z, \pi)$ with smallest modulus. For $\alpha=1$ this smallest modulus is approximately .707 .

\section{REFERENCES}

1. J. F. Paydon and H. S. Wall, The continued fraction as a sequence of linear transformations, Duke Math. J., 9 (1942), 360-372.

2. O. Perron, Uber ein Schlichtheitsschranke von James S. Thale, S. -B. Math. -Nat. Kl. Bayer. Akad. Wiss. 1956.

3. J. S. Thale, Univalence of continued fractions and Stieltjes transforms, Proc. Amer. Math. Soc., 7 (1956), 232-244.

4. H. S. Wall, Analytic theory of continued fractions, Van Nostrand, New York, 1948.

De PaUl University

NORTHWESTERN UNIVERSITY 



\section{PACIFIC JOURNAL OF MATHEMATICS}

\section{EDITORS}

David GILbarg

Stanford University

Stanford, California

\section{F. H. BRowneLL}

University of Washington

Seattle 5, Washington

\section{A. L. Whiteman}

University of Southern California Los Angeles 7, California

\section{J. PAIGe}

University of California

Los Angeles 24, California

\section{ASSOCIATE EDITORS}
E. F. BECKENBACH
T. M. CHERRY
D. DERRY

\author{
E. HEWITT \\ A. HORN \\ L. NACHBIN
}

\author{
M. OHTSUKA \\ H. L. ROYDEN \\ M. M. SCHIFFER
}

E. SPANIER

E. G. STRAUS

F. WOLF

\section{SUPPORTING INSTITUTIONS}

\author{
UNIVERSITY OF BRITISH COLUMBIA \\ CALIFORNIA INSTITUTE OF TECHNOLOGY \\ UNIVERSITY OF CALIFORNIA \\ MONTANA STATE UNIVERSITY \\ UNIVERSITY OF NEVADA \\ NEW MEXICO STATE UNIVERSITY \\ OREGON STATE COLLEGE \\ UNIVERSITY OF OREGON \\ OSAKA UNIVERSITY \\ UNIVERSITY OF SOUTHERN CALIFORNIA
}

\author{
STANFORD UNIVERSITY \\ UNIVERSITY OF TOKYO \\ UNIVERSITY OF UTAH \\ WASHINGTON STATE COLLEGE \\ UNIVERSITY OF WASHINGTON \\ AMERICAN MATHEMATICAL SOCIETY \\ CALIFORNIA RESEARCH CORPORATION \\ HUGHES AIRCRAFT COMPANY \\ SPACE TECHNOLOGY LABORATORIES \\ NAVAL ORDNANCE TEST STATION
}

\footnotetext{
Mathematical papers intended for publication in the Pacific Journal of Mathematics should be typewritten (double spaced), and the author should keep a complete copy. Manuscripts may be sent to any one of the four editors. All other communications to the editors should be addressed to the managing editor, L. J. Paige at the University of California, Los Angeles 24, California.

50 reprints per author of each article are furnished free of charge; additional copies may be obtained at cost in multiples of 50 .
}

The Pacific Journal of Mathematics is published quarterly, in March, June, September, and December. The price per volume (4 numbers) is $\$ 12.00$; single issues, $\$ 3.50$. Back numbers are available. Special price to individual faculty members of supporting institutions and to individual members of the American Mathematical Society: $\$ 4.00$ per volume; single issues, $\$ 1.25$.

Subscriptions, orders for back numbers, and changes of address should be sent to Pacific Journal of Mathematics, 2120 Oxford Street, Berkeley 4, California.

Printed at Kokusai Bunken Insatsusha (International Academic Printing Co., Ltd.), No. 6, 2-chome, Fujimi-cho, Chiyoda-ku, Tokyo, Japan.

PUBLISHED BY PACIFIC JOURNAL OF MATHEMATICS, A NON-PROFIT CORPORATION

The Supporting Institutions listed above contribute to the cost of publication of this Journal, but they are not owners or publishers and have no responsibility for its content or policies. 


\section{Pacific Journal of Mathematics}

\section{Vol. 10, No. $4 \quad$ December, 1960}

M. Altman, An optimum cubically convergent iterative method of inverting a linear bounded operator in Hilbert space . . . . . . . . . . . . . . . . . . . . . . . . . . 1107

Nesmith Cornett Ankeny, Criterion for rth power residuacity ................. 1115

Julius Rubin Blum and David Lee Hanson, On invariant probability measures I . . . . . 1125

Frank Featherstone Bonsall, Positive operators compact in an auxiliary topology ..... 1131

Billy Joe Boyer, Summability of derived conjugate series . . . . . . . . . . . . . . . . 1139

Delmar L. Boyer, A note on a problem of Fuchs . . . . . . . . . . . . . . . . . 1147

Hans-Joachim Bremermann, The envelopes of holomorphy of tube domains in infinite

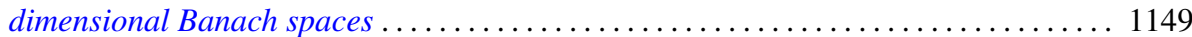

Andrew Michael Bruckner, Minimal superadditive extensions of superadditive

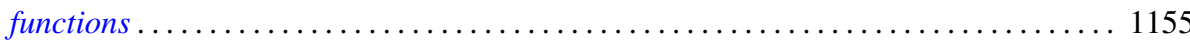

Billy Finney Bryant, On expansive homeomorphisms .................... 1163

Jean W. Butler, On complete and independent sets of operations in finite algebras . . . . . 1169

Lucien Le Cam, An approximation theorem for the Poisson binomial distribution ...... 1181

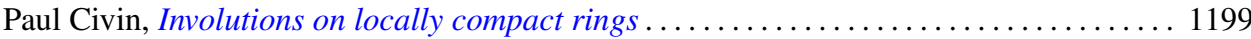

Earl A. Coddington, Normal extensions of formally normal operators . . . . . . . . . 1203

Jacob Feldman, Some classes of equivalent Gaussian processes on an interval ........ 1211

Shaul Foguel, Weak and strong convergence for Markov processes . . . . . . . . . . . 1221

Martin Fox, Some zero sum two-person games with moves in the unit interval ........ 1235

Robert Pertsch Gilbert, Singularities of three-dimensional harmonic functions . . . . . . . 1243

Branko Grünbaum, Partitions of mass-distributions and of convex bodies by

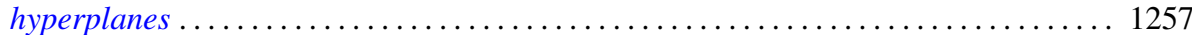

Sidney Morris Harmon, Regular covering surfaces of Riemann surfaces ........... 1263

Edwin Hewitt and Herbert S. Zuckerman, The multiplicative semigroup of integers

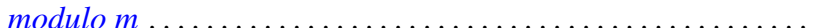

Paul Daniel Hill, Relation of a direct limit group to associated vector groups . ......... 1309

Calvin Virgil Holmes, Commutator groups of monomial groups . .

James Fredrik Jakobsen and W. R. Utz, The non-existence of expansive homeomorphisms

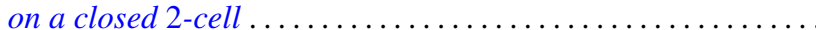

John William Jewett, Multiplication on classes of pseudo-analytic functions . . . . . . . 1323

Helmut Klingen, Analytic automorphisms of bounded symmetric complex domains . . . . 1327

Robert Jacob Koch, Ordered semigroups in partially ordered semigroups . . . . . . . . 1333

Marvin David Marcus and N. A. Khan, On a commutator result of Taussky and

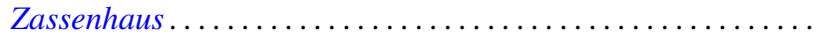

John Glen Marica and Steve Jerome Bryant, Unary algebras......

Edward Peter Merkes and W. T. Scott, On univalence of a continued fraction . . . . . . . 1361

Shu-Teh Chen Moy, Asymptotic properties of derivatives of stationary measures . . . . . 1371

John William Neuberger, Concerning boundary value problems . . . . . . . . . . . 1385

Edward C. Posner, Integral closure of differential rings . . . . . . . . . . . . . . . . . 1393

Marian Reichaw-Reichbach, Some theorems on mappings onto . . . . . . . . . . . . . 1397

Marvin Rosenblum and Harold Widom, Two extremal problems . . . . . . . . . . . . . . . . 1409

Morton Lincoln Slater and Herbert S. Wilf, A class of linear differential-difference

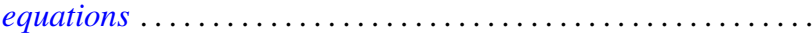

Charles Robson Storey, Jr., The structure of threads . . . . . . . . . . . . . . . . . . 1429

J. François Treves, An estimate for differential polynomials in $\partial / \partial z_{1},, \cdots, \partial / \partial z_{-} n \ldots \ldots 1447$

J. D. Weston, On the representation of operators by convolutions integrals . . . . . . . . 1453

James Victor Whittaker, Normal subgroups of some homeomorphism groups ......... 1469 